\title{
Emoções no cyberbullying: um estudo com adolescentes portugueses'
}

\author{
Ana Paula Caetano" \\ Isabel Freire" \\ Ana Margarida Veiga Simão"II \\ Maria José D. Martins ${ }^{\text {IV }}$ \\ Maria Teresa Pessoav
}

I- Este projeto, que integra investigadores das Universidades de Coimbra e de Lisboa e tem como investigador responsável João Amado, foi financiado no âmbito do Programa Operacional Temático Fatores de Competitividade (COMPETE) e coparticipado pelo Fundo Comunitário Europeu FEDER e pela Fundação para a Ciência e Tecnologia (Ref. PTDC/CPE-CED/108563/2008). Agradece-se a Sidclay Bezerra Sousa 0 apoio na revisão do texto quanto à forma.

II- Instituto de Educação, Universidade de Lisboa, Portugal.

Contato: apcaetano@ie.ulisboa.pt;

isafrei@ie.ulisboa.pt

III- Faculdade de Psicologia, Universidade de Lisboa, Portugal.

Contato: amsimao@psicologia.ulisboa.pt

IV- Escola Superior de Educação, Instituto Politécnico de Portalegre, Portugal.

Contato: mariajmartins@esep.pt

V- Faculdade de Psicologia e de Ciências da Educação, Universidade de Coimbra, Portugal.

Contato: tpessoa@fpce.uc.pt

\section{Resumo}

Neste artigo, apresenta-se uma parte do estudo extensivo do projeto Cyberbullying - um diagnóstico da situação em Portugal, com a aplicação de um questionário a 3.525 adolescentes no $6 .^{\circ}, 8 .^{\circ}$ e $11 .^{\circ}$ níveis de escolaridade. Com vista à caracterização do cyberbullying, pretendeu-se contribuir para a identificação e interpretação das emoções experienciadas pelos jovens envolvidos, quer como vítimas, quer como agressores em situações de cyberbullying. Os dados revelam que a tristeza, a vontade de vingança e o medo são as emoções mais frequentes das vítimas, enquanto a satisfação, a indiferença e o alívio são aquelas que os agressores mais vivenciam. Revelam ainda diferenças significativas entre as emoções experienciadas pelas vítimas e aquelas que os agressores lhes atribuem, o que, podendo ser um indicador da falta de empatia destes últimos, requer o desenvolvimento de uma educação emocional dos jovens. Verificam-se algumas diferenças significativas em relação ao nível de escolaridade, sexo, escola e município, nomeadamente: na maior incidência de emoções como a tristeza, o medo, a insegurança e a vontade de vingança nas vítimas do sexo masculino; mais sentimentos de insegurança, de alívio, de confusão e desorientação nas jovens agressoras; emoções associadas a impotência e falta de apoio, experimentadas pelos jovens no papel de vítimas, mais numas escolas do que noutras. Esses dados levam-nos a refletir sobre formas de agir no sentido da prevenção do cyberbullying.

\section{Palavras-chave}

Emoções - Cyberbullying - Emoções morais - Competências emocionais. 


\title{
Emotions in Cyberbullying: A study with Portuguese teenagers
}

\author{
Ana Paula Caetano" \\ Isabel Freire" \\ Ana Margarida Veiga Simão"II \\ Maria José D. Martins ${ }^{\mathrm{IV}}$ \\ Maria Teresa Pessoav
}

I- This project, which includes researchers from the Universities of Coimbra and Lisbon, and has as investigator-in-charge João Amado, was funded under the Thematic Operational Program Factors of Competitiveness (COMPETE - Programa Operacional Temático Fatores de Competitividade) and co-reimbursed by the European Community Fund FEDER and by the Foundation for Technology and Science (FCT - Fundação para a Ciência e Tecnologia), (Ref. PTDC / CPE-CED / 108563/2008).

II- Instituto de Educação, Universidade de Lisboa, Portugal.

Contact: apcaetano@ie.ulisboa.pt;

isafrei@ie.ulisboa.pt

III- Faculdade de Psicologia, Universidade de Lisboa, Portugal.

Contact: amsimao@psicologia.ulisboa.pt

IV- Escola Superior de Educação, Instituto Politécnico de Portalegre, Portugal. Contact: mariajmartins@esep.pt

V- Faculdade de Psicologia e de Ciências da Educação, Universidade de Coimbra, Portugal. Contact: tpessoa@fpce.uc.pt

\begin{abstract}
This paper presents a part of the extensive study of the project Cyberbullying - A Diagnosis of the Situation in Portugal, which applied a questionnaire to 3,525 teenagers in the 6 th, 8th and 11th schooling levels. In order to characterize cyberbullying, we have sought to contribute to the identification and interpretation of emotions experienced by the youngsters involved as victims or offenders in cyberbullying situations. The data reveal that sadness, desire for revenge, and fear are the most frequent emotions of the victims, while satisfaction, indifference and relief are those that the offenders experience the most. Data also reveal significant differences between the emotions experienced by victims and those that offenders attributed to them, which can be an indicator of the lack of empathy of the latter and thus requires the development of an emotional education of youngsters. Some significant differences have been found concerning schooling level, sex, school, and municipality, namely: a higher incidence of emotions such as sadness, fear, insecurity, and the desire for revenge among male victims; more feelings of insecurity, relief, confusion, and disorientation among young female offenders; emotions associated with impotence and lack of support experienced by youngsters in the role of victims, more in some schools than in others. These data lead us to reflect on how to take action to prevent cyberbullying.
\end{abstract}

\section{Keywords}

Emotions - Cyberbullying - Moral emotions - Emotional competencies. 


\section{Introdução}

0 bullying entre crianças e jovens é uma preocupação desde há muito tempo. Tem havido muita investigação internacional sobre $\mathrm{o}$ assunto $\mathrm{e}$ um aumento do conhecimento sobre a sua natureza e efeitos. Há também uma crescente experiência da eficácia de uma série de estratégias de intervenção em meio escolar (CASSIDY; FAUCHER; JACKSON, 2013; MENESINI; NOCENTINNI; PALADINO, 2012). Com o advento das novas tecnologias de informação e comunicação, surgiram novas formas de bullying, geralmente designadas de cyberbullying, com base no uso de ferramentas tecnológicas para maltratar sistematicamente outros. 0 cyberbullying é uma forma de agressão muitas vezes mais insidiosa que o bullying tradicional. 0 ambiente tecnológico faz com que as relações e os ambientes sociais sejam mais complexos, devido à expansão do espaço e do tempo, e isso faz com que a desigualdade de poder entre vítimas e perpetradores seja incomparavelmente maior (AMADO et al., 2009). A pesquisa mostra que as experiências sistemáticas de bullying têm um enorme impacto negativo sobre o desenvolvimento de seus protagonistas (SAHIN; AYDIN; SARI, 2012; SMITH; BRAIN, 2000; OLWEUS, 1993). São ainda poucos, no entanto, os estudos sobre as emoções experienciadas pelos protagonistas do cyberbullying, quer quando desempenham o papel de vítimas, quer quando desempenham o de agressores (ORTEGA; ELIPE; CALMAESTRE, 2009b).

Em experiências de vitimização e agressão, os estudos mostram que as vítimas apresentam frequentes sentimentos de vulnerabilidade, medo ou vergonha (BERAN; LI, 2005; DE MORAES BANDEIRA; HUTZ, 2010), de raiva e de desejo de vingança (BERAN; LI, 2005; DE MORAES BANDEIRA; HUTZ, 2010; ORTEGA; ELIPE; CALMAESTRE, 2009; ORTEGA et al., 2012; WILTON; CRAIG; PEPLER, 2000), como também de tristeza (ANDERSON; HUNTER, 2012; BERAN; LI, 2005; DE MORAES BANDEIRA; HUTZ, 2010; WILTON; CRAIG; PEPLER, 2000). Por outro lado, os agressores sentem prazer e satisfação em dominar, desenvolvem sentimentos de culpa e vergonha pelos atos praticados (DE MORAES BANDEIRA;
HUTZ, 2010), ou apresentam sentimentos de indiferença (BORG, 1998; WILTON; CRAIG; PEPLER, 2000; DE MORAES BANDEIRA; HUTZ, 2010).

Neste artigo, pretendemos evidenciar os resultados do estudo no que respeita às emoções experienciadas por jovens em situações de cyberbullying, quer enquanto vítimas quer enquanto agressores, bem como relacionar essas emoções com algumas variáveis sociodemográficas.

\section{Emoções - dos conceitos e teoria aos} estudos sobre bullying e cyberbullying

Entendemos aqui por emoções não apenas os programas complexos, em grande medida automatizados, de ações experienciadas e sentidas no nosso corpo, mas também os sentimentos de emoção que são as percepções compostas de tudo o que acontece no corpo e na mente quando sentimos emoções - incluindo as ações, as ideias e o estilo com que as ideias fluem (DAMÁSIO, 2010). Outros autores utilizam diferentes designações, tais como a de estados emocionais, que incluem essas componentes fisiológicas, cognitivas e comportamentais. Aqui, os sentimentos e as sensações correspondem às dimensões subjetivas. A figura 1 , que identifica as componentes das emoções, segundo Berthoz e Krauth-Gruber (2011), dá conta das dimensões subjetiva e motivacional a que daremos particular atenção neste artigo.

Figura 1 - Componentes das emoções

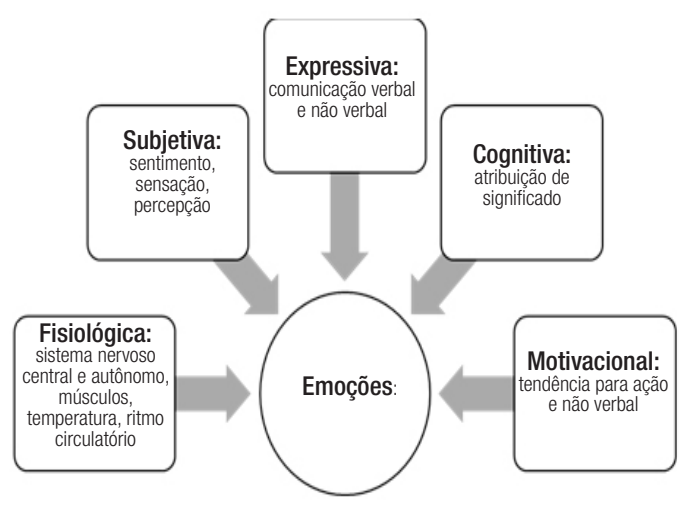

Fonte: Adaptado de Berthoz e Krauth-Gruber (2011). 
Analisaremos as emoções de acordo com várias classificações: positivas ou negativas, primárias ou secundárias (EKMAN, 2003). Algumas são entendidas como universais - as emoções primárias -, tais como alegria, tristeza, raiva e medo. Entre as emoções secundárias, derivadas de combinações de emoções primárias, estão culpa, arrependimento, desespero, insegurança, alívio, terror, humilhação (DAMÁSIO, 2001; EKMAN, 2003; MARTINS, 2009).

Na análise dos nossos dados, centrar-nos-emos nas emoções morais que acompanham as experiências de cyberbullying, partindo de uma teoria atribucional que distingue essas emoções quanto à atribuição que os sujeitos fazem acerca da possibilidade de controlar (ou não) as causas de uma determinada situação e de mudar as causas voluntariamente (WEINER, 2007).

Para a interpretação dos dados, utilizaremos uma outra proposta de autores que, num quadro conceitual integrador, apresentam uma teoria atribucional das emoções - a teoria do controle-valor das emoções, que estuda as emoções analisando se os indivíduos sentem que controlam ou que não controlam atividades e resultados que são significativos para eles (PEKRUN; FRENZEL; GOETZ, 2007).

As emoções parecem ser constituídas do conjunto das nossas experiências de valor, embora aquelas não sejam suficientes para fundar os valores, o que não significa dizer que estejam sempre presentes em todas essas experiências (LIVET, 2002). Algumas dessas emoções são particularmente relevantes no julgamento e decisão moral: são as chamadas emoções morais. Essas emoções, por si só, são objetos de julgamento, pois são socialmente consideradas certas ou erradas. Sendo normalmente dirigidas aos outros, servem não apenas para nos alertar, ler os estados emocionais, motivos e intenções dos outros, ajudar-nos a nos posicionar e a regular o comportamento social, de uma forma rápida e intuitiva, mas também para apoiar uma reflexão mais aprofundada acerca do sentido ético das situações, dos outros e das ações.

Neste estudo, aprofundamos algumas emoções morais, questionando sobre a componente subjetiva - sentimentos, percepções, sensações, expectativas e atribuições -, à semelhança de outros autores (ORTEGA; ELIPE; CALMAESTRE, 2009), relativas a culpa, injustiça, arrependimento, humilhação e zanga consigo próprio. Na sequência de outros estudos (PERREN et al., 2012), entendemos como emoções amorais as emoções positivas e neutras vividas em situações de sofrimento como o cyberbullying, aqui integrando emoções de satisfação, prazer, divertimento e do sentir-se mais forte.

Em alguns desses estudos, há uma fraca relação entre bullying e emoções morais, quer por aqueles que têm papel de vítimas, quer por aqueles que exercem o papel de agressores. No estudo de Perren et al. (2012), feito com adolescentes e usando medidas de produção face a uma situação hipotética de violação de regras morais, os participantes categorizados como agressores referem, a um nível bivariado, emoções amorais como orgulho, indiferença e felicidade, embora esse efeito tenha desaparecido num nível multivariado. Nesse estudo, parece haver relação negativa entre bullying e emoções morais (como vergonha, culpa e remorso), resultado semelhante àquele do estudo de Gasser e Keller (2009), realizado com crianças (de 7-8 anos), em que os agressores parecem ter menos tendência a dar razões morais às suas atribuições emocionais. Tal aspecto também é evidenciado no estudo feito por Menesini et al. (2003), em que os agressores atribuem mais indiferença e orgulho do que as vítimas e as crianças não envolvidas.

Essas relações negativas entre agressão e emoções morais também foram verificadas em outros estudos com adolescentes (ARSENIO; ADAM; GOLD, 2009; MENESINI; CAMODECA, 2008; TTOFI; FARRINGTON, 2008). A menor incidência de emoções morais associa-se a justificações morais, que apontam para menos comprometimento e responsabilidade moral, quer em crianças, quer em adolescentes (PERREN et al., 2012).

\section{Metodologia da investigação}

Como foi dito, este estudo faz parte de um projeto de investigação mais amplo, que se desenvolveu em duas etapas. Na primeira, de carácter exploratório, foi utilizado um questionário com 
perguntas de resposta aberta, aplicado a uma amostra de 339 estudantes do sexo masculino (168) e feminino (171), do $6^{\circ}, 8^{\circ}$ e $11^{\circ}$ ano de escolaridade, de sete escolas das zonas de Coimbra e de Lisboa, depois das necessárias autorizações do Ministério da Educação, da direção das escolas e das famílias. Na segunda, procedeu-se a um estudo mais extensivo, com aplicação de um questionário com perguntas de respostas mistas. É nessa última fase que se inscreve o estudo aqui apresentado. Uma cuidadosa e extensa revisão da literatura foi realizada ao longo do projeto, incluindo a consulta de instrumentos de coleta de dados usados na investigação da problemática do cyberbullying (e.g., ORTEGA, CALMAESTRA; MORA-MERCHÁN, 2007).

\section{Instrumento de investigação e procedimentos de coleta e de análise}

A análise de conteúdo dos dados da primeira etapa (AMADO et al., 2012) proporcionou a construção de conjuntos de indicadores que constituíram os itens das perguntas de resposta fechada do questionário da segunda etapa - Questionário de Diagnóstico sobre o Cyberbullying (QDC) -, que inclui quatro partes distintas. No início do questionário, é dada uma explicação sobre o que é o cyberbullying ${ }^{1}$, de forma que os participantes possam responder com base numa defınição comum. Seguem-se algumas questões destinadas a caracterizar a amostra em termos sociodemográficos (idade, sexo, local em que reside, profissão e nível acadêmico dos pais) e questões genéricas sobre a utilização das novas tecnologias. A segunda parte é constituída por conjuntos de questões que visam a coletar informações sobre os jovens no papel de vítima, e a terceira, por perguntas sobre os jovens no papel de agressores. Nessas duas partes do questionário, incluem-se questões sobre as emoções experienciadas no cyberbullying

I- A explicação dada no início do questionário é a seguinte: "As crianças e os jovens utilizam diariamente as novas tecnologias de comunicação e isso traz-Ihes grandes vantagens, mas também existem situações em que, através desses meios, alguns colegas maltratam outros com injúrias, calúnias, difamações, perseguições, através de mensagens por telefones móveis, por e-mail, num chat, no Hi5 etc. Estamos estudando esse fenômeno que se chama cyberbullying [...]". enquanto vítimas e enquanto agressores, bem como as emoções atribuídas pelas vítimas aos seus agressores. 0 quadro 2 apresenta a questão sobre emoções dos agressores.

Quadro 2 - Exemplo de pergunta do questionário - Emoções dos agressores

\begin{tabular}{|c|c|}
\hline \multicolumn{2}{|c|}{$\begin{array}{l}\text { 33. } 0 \text { que sentiste quando fizeste isso? } \\
\text { (assinala com uma cruz até } \mathbf{3} \text { opções) }\end{array}$} \\
\hline 33.1. Senti-me satisfeito/a & 33.9. Senti indiferença \\
\hline 33.2. Senti medo & $\begin{array}{l}\text { 33.10. Senti-me } \\
\text { arrependido/a }\end{array}$ \\
\hline 33.3. Senti prazer nisso & $\begin{array}{l}\text { 33.11. Senti que era divertido } \\
\text { fazer o que fiz }\end{array}$ \\
\hline 33.4. Senti-me triste & $\begin{array}{l}\text { 33.12. Senti vontade de não } \\
\text { ver ninguém }\end{array}$ \\
\hline $\begin{array}{l}\text { 33.5. Senti-me zangado/a } \\
\text { comigo }\end{array}$ & $\begin{array}{l}\text { 33.13. Senti-me admirado/a } \\
\text { pelos outros }\end{array}$ \\
\hline 33.6. Senti-me aliviado/a & 33.14. Senti-me culpado/a \\
\hline 33.7. Senti insegurança & $\begin{array}{l}\text { 33.15. Senti-me confuso/a e } \\
\text { perdido/a }\end{array}$ \\
\hline 33.8. Senti-me mais forte & 33.16. Senti-me aterrorizado \\
\hline 33.17. Outro sentimer & \\
\hline
\end{tabular}

Fonte: dados da pesquisa

Finalmente, a quarta parte do questionário é constituída por questões com as quais se pretende perceber os apoios a que os jovens recorrem nessas situações e inclui uma escala de ambiente escolar e outra de ambiente familiar.

Tanto o questionário exploratório como a sua versão final foram construídos pela equipe do projeto, constituída por investigadores especialistas em educação, em psicologia e em tecnologias da informação e comunicação, com ampla experiência de formação de professores no tema. A aplicação do questionário exploratório junto a 339 adolescentes dos mesmos níveis etários e de escolaridade da amostra selecionada para o questionário extensivo foi feita presencialmente por professores em formação com os especialistas da equipe e houve o cuidado de coletar informação que garantisse a adequação da linguagem ao nível etário e de escolaridade.

Também o questionário final foi aplicado presencialmente, de março a maio de 2012, com a colaboração direta de 23 professores das respectivas escolas, muitos dos quais tinham participado de um curso de formação promovido pelos investigadores membros do projeto. Sempre que 
possível, a aplicação fez-se na aula de formação cívica. 0 tempo de resposta dos alunos ao questionário variou de vinte a trinta minutos. Foram cumpridas todas as formalidades e ações necessárias e exigidas a uma investigação rigorosa e ética, incluindo a confidencialidade e o consentimento informado dos jovens participantes e dos pais dos menores, bem como a aceitação do estudo e de aplicação do questionário pelo Ministério da Educação, como referido anteriormente.

Neste artigo, apresentamos os resultados da análise relativos às emoções, relacionando-os com alguns dados sociodemográficos e com os motivos dos agressores (os motivos atribuídos pelas suas vitimas e os apontados pelos próprios agressores). Para além das análises estatísticas de caráter descritivo, foram utilizados testes de caráter inferencial, como os testes de quiquadrado, correlação de Spearman e Bynary Logistic Regression.

\section{Amostra}

0 questionário foi aplicado em 23 agrupamentos de escolas situadas nas regiões norte, centro e sul do país. Coletaram-se 3.525 respostas de alunos do ensino básico e secundário $\left(6^{\circ}\right.$, $8^{\circ}$ e $11^{\circ}$ ano), sendo 1.683 (cerca de $47,8 \%$ ) do sexo masculino e 1.837 (52,1\%) do sexo feminino, cuja distribuição é apresentada na tabela 1 . As idades variavam entre os 10 e os 23 anos, situando-se o seu valor médio nos 13,6 anos, com um desvio padrão de 2,3. Os valores da mediana e da moda situavam-se ambos nos 13 anos.

Tabela 1 - Amostra por sexo e ano de escolaridade

\begin{tabular}{|c|c|c|c|}
\hline & Masculino & Feminino & Total \\
\hline $6^{\circ}$ ano & $\begin{array}{c}592 \\
(16.8 \%)\end{array}$ & $\begin{array}{c}529 \\
(15 \%)\end{array}$ & $\begin{array}{c}1.121 \\
(31.8 \%)\end{array}$ \\
\hline & & & \\
\hline $8^{\circ}$ ano & $\begin{array}{c}588 \\
(16,7 \%) \\
\end{array}$ & $\begin{array}{c}628 \\
(17,8 \%)\end{array}$ & $\begin{array}{c}1.216 \\
(34,5 \%) \\
\end{array}$ \\
\hline $11^{\circ}$ ano & $\begin{array}{c}496 \\
(14,1 \%) \\
\end{array}$ & $\begin{array}{c}676 \\
(19,2) \\
\end{array}$ & $\begin{array}{c}1.172 \\
(33,2 \%) \\
\end{array}$ \\
\hline Missing data* & \multicolumn{2}{|c|}{$\begin{array}{c}16 \\
(0,4 \%)\end{array}$} & $\begin{array}{c}16 \\
(0,4 \%)\end{array}$ \\
\hline Total & $\begin{array}{c}1676 \\
(47,6 \%)\end{array}$ & $\begin{array}{l}1833 \\
(52 \%)\end{array}$ & $\begin{array}{l}3.525 \\
(100 \%)\end{array}$ \\
\hline
\end{tabular}

* Cinco participantes não mencionaram o seu gênero e onze não indicaram o nível de escolaridade. Fonte: Dados da pesquisa.
Questionados sobre se alguma vez, durante o último ano, tinham sido vítimas e/ ou agressores de ofensas, difamações, ameaças, perseguições, através do telefone celular ou da internet, 7.6\% ( $\mathrm{N}=267)$ dos sujeitos responderam que tinham sido vítimas e 3.9\% $(\mathrm{N}=138)$ dos jovens responderam ter sido agressores de cyberbullying. No quadro 3, apresenta-se uma caracterização das vítimas e agressores de cyberbullying, levando em consideração o gênero e o ano de escolaridade.

Tabela 2 - Distribuição da amostra por nível de escolaridade e por gênero

\begin{tabular}{l:c:ccccc} 
& $\mathrm{M}$ & Vítimas & Total & $\mathrm{M}$ & $\mathrm{F}$ & Total \\
\hline $6^{\circ}$ ano & 28 & $\mathbf{3 3}$ & 61 & $\mathbf{1 4}$ & 4 & 18 \\
\hline $8^{\circ}$ ano & 29 & $\mathbf{5 0}$ & 79 & $\mathbf{2 3}$ & 16 & 39 \\
\hline $11^{\circ}$ ano & 43 & $\mathbf{8 3}$ & 126 & $\mathbf{4 5}$ & 36 & 81 \\
\hline Total & 100 & 166 & $266^{\star}$ & 82 & 56 & 138 \\
\hline
\end{tabular}

Nos dados apresentados na tabela 2, é possível observar, na distribuição da amostra, relativamente aos três anos de escolaridade, um número maior de vítimas do sexo feminino, ao mesmo tempo em que se verificou uma maior ocorrência relativamente ao sexo masculino nos casos de agressões. A idade média das vítimas é de 14,35 anos e a dos agressores é de 15,01 anos, o que indica uma idade média mais elevada do que a da amostra global, sendo a idade média dos agressores mais elevada que a das vítimas. Mais à frente, analisamos a relação entre essas variáveis sociodemográficas e as emoções.

\section{Resultados}

\section{Emoções das vítimas}

Apresentamos, na tabela 3, o conjunto dos dados relativos às emoções assinaladas pelas vítimas (as quais deveriam escolher três de um conjunto de quinze), e àquelas atribuídas pelos agressores às vítimas como tendo sido sentidas na situação que durante o último ano consideraram mais grave. 
Tabela 3 - Emoções das vítimas referidas pelas próprias e pelos agressores

\begin{tabular}{|c|c|c|}
\hline Emoção das vítimas & $\begin{array}{l}\text { Referidas pelas } \\
\text { vítimas } \\
\mathrm{N}=267\end{array}$ & $\begin{array}{c}\text { Referidas pelos } \\
\text { agressores } \\
\mathrm{N}=138\end{array}$ \\
\hline Sentir tristeza & $44,9 \%$ & $18,7 \%$ \\
\hline Sentir raiva & $39,5 \%$ & $27,6 \%$ \\
\hline Sentir vontade de vingança & $35,8 \%$ & $32,1 \%$ \\
\hline Sentir medo & $23,9 \%$ & $24,6 \%$ \\
\hline Sentir humilhação & $23,6 \%$ & $33,6 \%$ \\
\hline Sentir indiferença & $20,7 \%$ & $19,4 \%$ \\
\hline Sentir vontade de fugir & $19.9 \%$ & $10,4 \%$ \\
\hline Sentir insegurança & $19.9 \%$ & $13,4 \%$ \\
\hline Sentir estar confuso e perdido & $17 \%$ & $9,7 \%$ \\
\hline $\begin{array}{l}\text { Sentir vontade de não ver } \\
\text { ninguém }\end{array}$ & $16,6 \%$ & $5,2 \%$ \\
\hline Sentir estar sozinho e indefeso & $14,4 \%$ & $9 \%$ \\
\hline Sentir injustiça & $11,8 \%$ & $9,7 \%$ \\
\hline Sentir terror & $10,3 \%$ & $5,2 \%$ \\
\hline Sentir desespero & $9,6 \%$ & $6,0 \%$ \\
\hline Sentir culpa & $2,2 \%$ & $25,4 \%$ \\
\hline
\end{tabular}

Fonte: dados da pesquisa

Como se pode ver na tabela 3 , as cinco emoções mais referidas são, todas elas e como era expectável, emoções negativas, associadas a sofrimento; e, em $6^{\circ}$ lugar, surge a indiferença. Destaca-se a tristeza como a emoção mais presente (em quase 50\% dos respondentes que referiram situações no papel de vítimas). Trata-se de uma emoção não ativa, introjetada, com um grau de intensidade relativamente menor do que outras, como o desespero $(9,6 \%)$ e o terror $(10,3 \%)$, bem menos frequentes, embora de não menos importância, pela sua força, e que parecem apontar para um sentimento de falta de controle sobre a situação (PEKRUN et al., 2007).

Por contraposição, surgem frequentemente, logo a seguir à tristeza, em termos de percentagem, emoções negativas que apelam à ação, como a raiva $(39,5 \%)$ e a vontade de se vingar (35,8\%). 0 medo $(24,6 \%)$ e a insegurança $(19,9 \%)$ são também manifestações que podem estar associadas a um sentimento de falta de controle sobre as situações, embora referidas numa frequência inferior. No mesmo sentido, surgem $14,4 \%$ de referências ao sentir-se so- zinho e indefeso e emoções que orientam para o evitamento, como o sentir vontade de fugir $(19,9 \%)$ e vontade de não ver ninguém $(16,6 \%)$. A essas respostas não será alheio o fato de muitas vezes o cyberbullying estar associado a imagens que permanecem no tempo, em espaço virtual. Talvez tais respostas sejam também associáveis ao fato de as vítimas, geralmente muito vulneráveis do ponto de vista da autoestima, verem essa situação agravada depois de vitimadas, o que tende a diminuir a sua capacidade de relacionamento com os outros.

Ainda em relação às emoções das vítimas - o sentimento de transgressão moral pelos agressores -, evidenciam-se emoções como a humilhação $(23,6 \%)$ e a injustiça $(11,8 \%)$. Em contrapartida, ainda a respeito desse nível moral, as vítimas muito raramente atribuem a si próprias sentimentos de culpa (2,2\%), não se reconhecendo responsáveis pelas situações vividas, ao contrário do que noutro tipo de situações de vitimização parece acontecer (GRAHAM; JUVONEN, 1998). Relativamente às emoções das vítimas referidas pelos agressores, destacam-se humilhação (33,6\%), vontade de vingança $(32,1 \%)$, raiva $(27,6 \%)$, culpa $(25,4 \%)$ e medo $(24,6 \%)$.

\section{Emoções dos agressores}

No que respeita às emoções sentidas pelos próprios agressores, conforme distribuição apresentada na tabela 4, destacam-se as emoções ditas positivas, como a satisfação $(32,6 \%)$, o alívio (26,5\%), a diversão $(18,2 \%)$, o prazer $(19,7 \%)$, o sentir-se mais forte $(14,4 \%)$, emoções que parecem remeter para interesses egóicos e para um sentido de controle sobre as situações.

Ressaltamos que esses tipos de emoções referidas e sentidas pelos agressores surgem também em estudos com adolescentes sobre agressão entre pares, em contextos face a face; esses adolescentes invocavam o divertimento e a brincadeira como os motivos mais frequentes que utilizavam para justificar a agressão, mas que nem sempre eram partilhados pelas vítimas e observadores da mesma (MARTINS, 2013). 
Tabela 4 - Emoções dos agressores ( $N=138)$

\begin{tabular}{|c|c|c|}
\hline \multirow{2}{*}{ Emoções dos agressores } & \multicolumn{2}{|c|}{ Respostas dos agressores } \\
\hline & $f$ & $\%$ \\
\hline Senti-me satisfeito & 43 & 32,6 \\
\hline Senti indiferença & 37 & 28 \\
\hline Senti-me aliviado & 35 & 26,5 \\
\hline Senti prazer & 26 & 19,7 \\
\hline Senti que era divertido & 24 & 18,2 \\
\hline Senti-me mais forte & 19 & 14,4 \\
\hline Senti-me arrependido & 18 & 13,6 \\
\hline Senti-me culpado & 14 & 10,6 \\
\hline Senti-me zangado comigo & 12 & 9,1 \\
\hline Senti-me triste & 9 & 6,8 \\
\hline Senti vontade de não ver ninguém & 8 & 6,1 \\
\hline Senti-me confuso e perdido & 4 & 3 \\
\hline Senti-me admirado & 4 & 3 \\
\hline Senti insegurança & 3 & 2,3 \\
\hline Senti medo & 1 & 0,8 \\
\hline Senti-me aterrorizado & 1 & 0,8 \\
\hline
\end{tabular}

A presença de $28 \%$ de respostas relativas à indiferença - a segunda emoção mais referida poderá indicar, mais uma vez, uma dificuldade de sentir empatia para com as vítimas face às situações de dor infligidas a terceiros. Corroborando essa tendência, são menos frequentes as emoções que poderiam assinalar algum sentido ético e autocrítico, embora não sejam de menosprezar respostas relativas ao sentimento de culpa, por $10,6 \%$ dos respondentes, ao arrependimento, por $13,6 \%$, ao sentir-se zangado consigo próprio, por 9,1\%, ou mesmo à vontade de não ver ninguém, por 6,1\% dos agressores. Esses dados são também similares ao que é encontrado em alguns estudos sobre agressão em contextos face a face (MARTINS, 2013).

\section{Comparação entre emoções das vítimas e emoções atribuídas pelos agressores às vítimas}

Comparando as emoções sentidas pelas vítimas com aquelas que lhes são atribuídas pelos agressores, é possivel encontrar convergências e divergências com interesse para reflexão (só 62 respondentes referem ter estado implicados em ambos os tipos de papéis).

Usando o teste de qui quadrado, emergem apenas três emoções onde há uma significância estatística, pelo que apenas em três emoções os respondentes são coerentes quando estão na posição de vítimas e agressores, na atribuição de emoções às vítimas: vontade de vingança $\left(\mathrm{x}^{2}\right.$ (g.l. $\left.)=12,74, \mathrm{p}<0,01\right)$; injustiça $\left(\mathrm{x}^{2}\right.$ (g.l.) $=$ 18,794, $p<0,01)$; sentir-se sozinho e indefeso $\left(\mathrm{x}^{2}\right.$ (g.l.) $\left.=6,3, \mathrm{p}<0,05\right)$.

Para todas as outras emoções, não há coerência ou a coerência não é suficientemente alta para se poder considerar estatisticamente significativa: medo ( $x^{2}$ (g.l.) $=2,304, p<0,05$ ), terror $\left(x^{2}\right.$ (g.l.) $\left.=2,018, p<0,05\right)$, vontade de fugir ( $x^{2}$ (g.l.) $\left.=1,896, p<0,05\right)$, vontade de não ver ninguém $\left(\mathrm{x}^{2}\right.$ (g.l.) $\left.=0,690, \mathrm{p}<0,05\right)$, culpa $\left(\mathrm{x}^{2}\right.$ (g.l.) $=0,296, p<0,05)$, confusão ( $x^{2}$ (g.l.) $=5,108$, $p<0,05)$, desespero ( $x^{2}$ (g.l.) $\left.=2,437, p<0,05\right)$, raiva $\left(x^{2}\right.$ (g.l. $\left.)=0,655, p<0,05\right)$, humilhação $\left(x^{2}\right.$ (g.l.) $=1,745, \mathrm{p}<0,05)$, tristeza $\left(\mathrm{x}^{2}\right.$ (g.l. $)=1,380$, $\mathrm{p}<0,05$ ), insegurança ( $x^{2}$ (g.l.) $\left.=0,030, p<0,05\right)$ e indiferença $\left(\mathrm{x}^{2}\right.$ (g.l.) $\left.=0.850, \mathrm{p}<0,05\right)$. Esses dados parecem denotar uma dificuldade de os agressores se colocarem no lugar das suas vítimas, muito embora sejam também vítimas noutras situações.

Uma análise estatística mais ampla, não inferencial, do conjunto das respostas de vítimas e agressores sobre emoções das vítimas pode ser feita a partir da tabela 3. Aqui aumenta o número total de respostas, pois ela inclui todas as situações e não apenas aquelas cujos respondentes assumem o duplo papel de vítimas e agressores.

De um modo geral, não há coincidência entre as respostas atribuídas às vítimas pelos agressores e as referidas pelas vítimas. Veja-se, a título de exemplo, as emoções em que essas diferenças são mais notórias. Em primeiro lugar, ressalta o sentimento de culpa que as vítimas raramente referem sentir (2,2\%), ao contrário do que pensam os agressores (25,4\%). Trata-se provavelmente de uma interpretação distinta que uns e outros fazem da situação, podendo significar uma tendência dos agressores a culpar as vítimas e a esperar que elas os reconheçam, enquanto as vítimas tendem a não se reconhecer como culpadas das situações vividas.

Saliente-se, ainda, o não reconhecimento por muitos agressores da emoção mais referida 
pelas vítimas - a tristeza (referido por 44,9\% das vítimas e reconhecido por $18,7 \%$ dos agressores) -, bem como a dificuldade de assumir as consequências emocionais mais intensas, como o terror (referido por 10,3\% das vítimas e 5,2\% dos agressores) e o desespero (referido por 9,6\% das vítimas e 6,0\% dos agressores). Essas diferenças podem significar a presença de um comportamento egocêntrico, dificuldade dos agressores apresentarem um comportamento empático e de perceberem até que ponto são negativas as consequências dos seus atos. Podem também significar, pelo menos para alguns, que se trata de um processo de negação para lidar com o estresse, minimizando o valor do outro e possíveis sentimentos de culpa, que emergiriam caso se sentissem responsáveis por tais consequências (BARRIGA; GIBBS, 1996; ORTEGA, et al., 2009).

Por outro lado, algumas emoções são referidas com frequência similar por agressores e vítimas, como é o caso do medo, da vontade de vingança e da insegurança, o que poderá significar que essas emoções são tendencialmente mais consensuais e mais facilmente reconhecidas por todos. É ainda interessante verificar a referência explícita (na pergunta de resposta aberta) às emoções associadas ao divertimento por oito agressores, por contraposição com apenas uma referência pelas vítimas. Mais uma vez, verifica-se uma tendência dos agressores minimizarem a gravidade dos seus comportamentos e atribuírem às vítimas as suas próprias emoções e motivos (em que a brincadeira e o divertimento sobressaem).

\section{Emoções e variáveis sociodemográficas}

Com base na utilização do coeficiente de Spearman, é possível analisar as emoções em que é significativa a correlação entre emoções, idade, nível de escolaridade e sexo.

No que respeita ao nível de escolaridade, não há relações significativas entre emoções, nível de escolaridade e idade, a não ser para sentir-se divertido, mais presente no caso dos agressores de nível de escolaridade mais elevada $(\mathrm{r}=-0,184 ; \mathrm{p}<0,05)$.
No que respeita ao sexo, verificam-se diferenças significativas relativas a algumas emoções. Emoções como a tristeza $(r=0,260 ; p$ $<0,01)$, o medo ( $r=0,173 ; p<0,01)$, a insegurança $(\mathrm{r}=0,188 ; \mathrm{p}<0,01)$ e a vontade de se vingar $(\mathrm{r}=$ $0,165 ; p<0,01)$ são mais frequentes nas vítimas do sexo masculino do que nas de sexo feminino. Nas agressões realizadas pelo sexo feminino, mais do que no sexo masculino, surgem referências a sentimentos de insegurança $(r=-0,178 ; p<0,05)$, a sentir-se aliviado $(r=-0,214 ; p<0,05)$ ou a sentir-se confuso e perdido ( $r=0,206 ; p<0,05)$. Esses dados parecem indiciar que as diferenças emocionais entre sexos são também função dos papéis que desempenham no cyberbullying, acentuando-se nas vítimas do sexo masculino e nos agressores do sexo feminino.

Continuando a análise de correlações, utilizando o coeficiente de Spearman, verificam-se algumas correlações interessantes entre emoções e escola, quer por parte do agressor quer por parte da vítima. Em relação ao agressor, há diferenças significativas entre escolas no que respeita ao sentimento de arrependimento. Em relação às vítimas, essa lista de emoções é mais extensa e inclui as seguintes respostas: senti-me aterrorizado $(\mathrm{r}=$ $0,129 ; \mathrm{p}<0,05)$; senti insegurança $(\mathrm{r}=0,151$; $\mathrm{p}<0,05)$; vontade de não ver ninguém ( $\mathrm{r}=$ 0,$173 ; p<0,01$ ); senti-me confuso e perdido ( $r$ $=0,126 ; \mathrm{p}<0,05)$.

Esses resultados apontam para a importância do éthos escolar e a sua dimensão moral, nas escolas onde é mais frequentemente referido, pelos agressores, o arrependimento.

No que respeita ao município, a relação é muito menos evidente para as emoções das vítimas - só surge em relação à vontade de não ver ninguém $(r=0,173$; $\mathrm{p}<0,01)$. Mas, para o caso dos agressores, há diferenças significativas entre municipios no que respeita ao sentimento de culpa ( $\mathrm{r}$ $=0,216 ; \mathrm{p}<0,05)$ e ao arrependimento $(\mathrm{r}=$ $0,242 ; p<0,01)$, o que poderá estar associado a culturas locais onde as dimensões morais e normativas são mais acentuadas. 


\section{Discussão e contributos de intervenção}

Em sintese, os dados apontam para a importância e diferente incidência que diversas emoções parecem ter na experiência de cyberbullying, variando consoante os papéis aí desempenhados. As vítimas tendem a sentir, com mais frequência, emoções como a tristeza, a vontade de vingança e o medo, enquanto os agressores tendem a sentir satisfação, indiferença, alívio e prazer, resultados estes também encontrados nos estudos realizados por De Moraes Bandeira e Hutz (2010) e Martins (2013). Verificam-se ainda algumas diferenças significativas em nível de sexo, escolaridade, escola e município.

Nas experiências emocionais, não se revelaram diferenças significativas em relação à idade e, no que respeita ao nível de escolaridade, apenas surge como significativa a diversão, mais frequente nos agressores de nível de escolaridade mais elevado. Já em relação ao sexo, observaram-se algumas diferenças significativas, nomeadamente na maior incidência de emoções como a tristeza, o medo, a insegurança e a vontade de vingança nas vítimas do sexo masculino. No papel de agressoras, as jovens dizem experienciar mais sentimentos de insegurança, alívio, confusão e desorientação do que os jovens.

A análise dos dados revela, ainda, diferenças significativas entre escolas, nomeadamente em emoções associadas à impotência e falta de apoio experimentadas pelos jovens no papel de vítimas. Existem também alguns indicadores de relações significativas entre a escola, o município e o modo como o fenômeno é experienciado emocionalmente pelos agressores, nomeadamente emoções de arrependimento e sentimentos de culpa, o que nos leva a questionar o papel das culturas locais. Esses resultados poderão indiciar diferenças entre as escolas na forma como nelas se lida com as situações de cyberbullying, apontando para a importância do éthos escolar e sua dimensão moral, em que o aprender a viver juntos e com os outros se faz importante, um dos maiores desafios da educação (DELORS et al., 1999). Assim, o arrependimento dos agressores, mais referido em algumas escolas do que em outras, poderá significar diferenças na responsabilização que nessas escolas e comunidades se faz, enquanto as diferenças quanto a sentimentos de terror, insegurança e confusão poderão indicar diferenças entre as escolas quanto à proteção e apoio prestado nessas situações de cyberbullying.

À semelhança de outros estudos, os estudantes têm percepções diferentes em função de seu papel (de vítima ou agressor). Por exemplo, no mesmo sentido do estudo de Beran e Li (2005), De Moraes Bandeira e Hutz (2010) e Ortega, Elipe e Calmaestre (2009), as vítimas afırmam em maior proporção sentir-se mal e tristes, com medo e vontade de vingança, e os agressores são menos sensíveis aos sentimentos de sofrimento, quer na percepção de si próprios, quer na atribuição de sentimentos às vítimas. Pelo contrário, as emoções mais frequentes dos agressores são positivas e amorais, como satisfação, alívio, prazer, divertimento, sentir-se forte, a par da indiferença e de motivos de diversão, de brincadeira e de não ter nada para fazer, tratando-se de um conjunto de indicadores que parecem apontar para uma orientação hedonista e egocêntrica (MENESINI et al., 2003; PERREN et al., 2012). No mesmo sentido, as emoções morais são menos frequentes quer nas vítimas, quer nos agressores, embora não sejam de menosprezar os dados relativos aos agressores sobre a presença de arrependimento, culpa, zanga consigo próprio e tristeza, que indicam um sentido moral e um julgamento de si próprio. Nas vítimas, a escassa presença de sentimentos de culpa parece estar em contracorrente com outros estudos nos quais as vítimas tendem a culpabilizar-se (GRAHAM; JUVONEN, 1998). Por outro lado, à semelhança de outras investigações, os dados apontam para uma dificuldade de os agressores serem empáticos com as vítimas, mesmo quando eles próprios também são vítimas (MALTI; PERREN; BUCHMANN, 2010; ORTEGA; ELIPE; CALAMAESTRE, 2009), e só claramente o conseguem no caso da vingança. 
0 cyberbullying é um fenômeno que está associado ao sofrimento, sobretudo ao daqueles que o vivem como vítimas, mas também de alguns dos que agridem, mesmo quando por brincadeira, bem como daqueles que com eles interagem. Os nossos dados apontam efetivamente para uma sensibilidade reduzida, por parte dos agressores, ao sofrimento. No contexto do ciberespaço, os indivíduos têm menos sinais afetivos, sociais e contextuais do que na presença dos outros (AOYAMA; BARNARD-BRAK; TALBERT, 2011, p. 4). No caso do cyberbullying, os agressores estarão mais protegidos, dado o anonimato de que se reveste esse comportamento, e desconhecem as reações das vítimas, o que "os protege" de sentimentos de culpa (PESSOA; AMAD0, no prelo). Aqui, não existe uma desaprovação social direta e o sofrimento da vítima não é visível (AOYAMA, BARNARD-BRAK; TALBERT, 2011), muito embora o sofrimento causado pelo bullying possa ser fator de perturbação psicológica, desordem, ansiedade e baixa autoestima (CAMPBELL et al., 2013; DE MORAES BANDEIRA; HUTZ, 2010; PATCHIN; HINDUJA, 2010; SAHIN; AYDIN; SARI, 2012).

Tendo em conta as dificuldades e emoções sentidas, quer pelos que se referem como vítimas, quer pelos que o fazem como agressores, é preciso não apenas equacionar uma intervenção que procure prevenir o cyberbullying e algumas das causas que lhe estão na origem, mas também quebrar o ciclo da violência que, por vezes, se desenvolve em ambos os sentidos.

Gerir emoções, ler emoções, usar emoções são competências emocionais relevantes para lidar com situações adversas. Competências usadas ao serviço de motivações que nos levam a evitar, aliviar, ampliar essas emoções. As emoções, só por si, têm funções adaptativas que nos ajudam a nos situarmos e que nos orientam rapidamente. Mas elas podem, ainda, bloquear o nosso discernimento e levar-nos ao desajuste. Tudo depende da intensidade, do modo como as expressamos, como as deixamos fluir ou nos cristalizamos em torno delas. Parece-nos, assim, importante considerá-las e investir no sentido de desenvolvimento dessas competências socioemocionais, integrando-as numa abordagem multidimensional do cyberbullying (MARTINS, 2009).

Trata-se de trabalhar competências socioemocionais (SAARNI, 2000; TOPPING; HOLMES; BREMNER, 2000) e éticas que ou estão deficitárias ou não são mobilizadas nas situações concretas (entre outros, por fatores motivacionais). Entre essas, parecem particularmente relevantes competências relacionadas com: a empatia e compreensão das emoções dos outros; a compreensão das suas próprias emoções e motivações; o lidar com fatores de estresse; a gestão das suas próprias emoções; o sentir que têm controle sobre seus próprios destinos; a resolução de conflitos; o respeito às diferenças; o fazer amigos, ter liderança e proatividade para lidar com e desencorajar o cyberbullying; a autoestima; o respeito; a responsabilidade.

Trata-se também de desenvolver uma efetiva cidadania digital através de uma educação que forme as crianças e os jovens para a utilização informada, consciente e crítica das novas tecnologias da informação e da comunicação.

Mas não basta um trabalho de formação de crianças e jovens. Também importa desenvolver um clima escolar positivo e uma cultura escolar colaborativa, mediadora e de corresponsabilidade, em que todos sintam que são parte da solução e que os seus atos têm consequências. 0 nosso estudo também mostra que as emoções estão relacionadas com as escolas, nomeadamente as emoções morais. Por isso, é preciso desenvolver projetos de intervenção baseados no conhecimento aprofundado do fenômeno, numa abordagem sistêmica em que todos são envolvidos.

Para fins de considerações, acentuamos a necessidade de promover a participação ativa dos alunos, nomeadamente nas políticas institucionais de prevenção, em debates, no dia a dia. Também é necessário o envolvimento dos pais e supervisão, com afeto, estimulando o diálogo com os filhos, não culpando as vítimas, não retirando a internet, mas não se demitindo do seu 
papel na educação de valores, por serem modelos e por encorajarem seus filhos a ajudar colegas. É urgente desenvolver esforços coordenados nas escolas, famílias e comunidades, criando redes e uma cultura de proximidade e participação.

Na mudança a operar, é importante perceber:

[...] que os agressores e as vítimas são partes intermutáveis do mesmo, a um tempo sujeitos e objetos, ambos presos na mesma armadilha e da qual só poderão ser resgatados quando se abrir o círculo. Um círculo onde os rótulos, os preconceitos, os julgamentos são escrutinados para poderem ser transmutados. Um círculo onde os próprios percebem que também há neles parte da responsabilidade de se libertarem. Um círculo onde se percebe que a dor não é só naqueles que se refugiam no silêncio. Um círculo onde é possível em conjunto procurar as respostas. Abrindo-se a uma dinâmica ela própria imprevisível, da qual não há garantias de quais serão os resultados. (SOUZA; VEIGA SIMÃO; CAETANO, 2014, p. 590).

\section{Referências}

AMADO, João et al. Cyberbullying: um desafio à investigação e à formação. Interacções, Santarém, v. 13, p. 301-326, 2009.

AMADO, João et al. 0 cyberbullying e a escola: uma análise da situação em Portugal. In: COLÓQUIO LUSO -BRASILEIRO DE SOCIOLOGIA DA EDUCAÇÃO, 3., Rio de Janeiro, 25-27 de julho de 2012. Atas do III Colóquio Luso -Brasileiro... Rio de Janeiro: [s. n.], 2012.

ANDERSON, Shayron; HUNTER, Simon. Cognitive appraisals, emotional reactions, and their associations with three forms of peer-victimization. Psicothema, Oviedo, v. 24, n. 4, p. 621-627, 2012.

AOYAMA, IKuko; BARNARD-BRAK, Lucy; TALBERT, Tony L. Cyberbullying among high school students: Cluster analysis of sex and age differences and the level of parental monitoring. International Journal of Cyber Behavior, Psychology and Learning, v. 1, n. 1, p. 25-35, 2011.

ARSENIO, William; ADAMS, Erin; GOLD, Jason. Social information processing, moral reasoning and emotion attributions. Relations with adolescence' reactive and proactive aggression. Child Development, Malden, v. 80, n. 6, p.1739-1755, nov./dez. 2009.

DE MORAES BANDEIRA, Cláudia; HUTZ, Claudio Simon. As implicações do bullying na auto-estima de adolescentes. Psicologia Escolar e Educacional, Campinas, v. 14, n. 1, p. 131-138, 2010.

BARRIGA, Alvaro; GIBBS, John. Measuring cognitive distortion in antisocial youth: development and preliminary validation of the "How I Think" questionnaire. Aggressive Behavior, Malden, v. 22, n. 5, p. 333-343, 1996.

BERAN, Tanya; LI, Qing. Cyber-Harassment: A study of a new method for an old behavior. Journal of Educational Computing Research, Manchester, v. 32, n. 3, p. 265-277, 2005.

BERTHOZ, Sylvie; KRAUTH-GRUBER, Sylvia. La face caché des émotions. Paris: Le Pommier: Universcience, 2011. 189 p.

BORG, Mark. The emotional reactions of school bullies and their victims. Educational Psychology, Oxford, v. 18, n. 4, p. 433-445, 1998.

CAMPBELL, Marilyn A. et al. Do cyberbullies suffer too? Cyberbullies' perceptions of the harm they cause to others and to their own mental health. School Psychology International, v. 34, n. 6, p. 613-629, Dec. 2013.

CASSIDY, Wanda; FAUCHER, Chantal; JACKSON, Margaret. Cyberbullying among youth: a comprehensive review of current international research and its implications and application to policy and practice. School Psychology International, v. 34, n. 6, p. 575-612, Dec. 2013.

DAMÁSIO, António. 0 erro de Descartes. Mem Martins. Lisboa: Europa-América. 2001. 380 p.

DAMÁSIO, António. 0 livro da consciência. Lisboa: Temas e Debates, 2010. 432 p.

DELORS, Jacques et al. Educação: um tesouro a descobrir. Relatório para a UNESCO da Comissão Internacional sobre Educação para o século XXI. São Paulo: Cortez, 1999. 288 p.

EKMAN, Paul. Qué dice ese gesto? Barcelona: Integral, 2003. 320 p. 
GASSER Luciano; KELLER, Monika. Are the competent the morally good? Perspective taking and moral motivation of children involved in bullying. Social Development, Malden, v. 18, n. 4, p. 198-816, nov. 2009.

GRAHAM, Sandra; JUVONEN, Jaana. Self blame and peer victimization in middle school: an attributional analysis. Developmental Psychology, Washington, v. 34, n. 3, p. 587-599, 1998.

LIVET, Pierre. Émotions et rationalité morale. Paris: PUF, 2002.

MALTI, Tina; PERREN, Sonja; BUCHMANN, Marlis. Children's peer victimization, empathy, and emotional symptoms. Child Psychiatry and Human Development, v. 41, n. 1, p. 98-113, Feb. 2010.

MARTINS, Maria José D. Agressão, vitimização e emoções na adolescência, em contexto escolar e de lazer. Escola Superior de Educação do Instituto Politécnico de Portalegre. Interações, Santarém, v. 13, p. 87-207, 2009.

MARTINS, Maria José D. Problemas relacionais na escola: explicações e sentimentos dos adolescentes. In: SERPA, Margarida; CALDEIRA, Susana; GOMES, Carlos. (Org.). Resolução de problemas em contexto escolar. Lisboa: Colibri, 2013. p.79-100.

MENESINI, Ercilia; CAMODECA, Marina. Shame and guilt as behaviour regulators: Relationships with bullying, victimization and prosocial behaviour. British Journal of Developmental Psychology, Malden, v. 26, n. 2, p.183-196, June 2008.

MENESINI, Ercilia; NOCENTINNI, Annalaura.; PALADINO, Benedetta Emanuela. Empowering students against bullying and cyberbullying: evaluation of an Italian peer-led model. International Journal of Conflict and Violence, Bielefeld, v. 6, n. 2, p.314-321, Dec. 2012.

MENESINI, Ercilia et al. Moral emotions and bullying: a cross-national comparison of differences between bullies, victims and outsiders. Aggressive Behavior, Malden, v. 29, n. 6, p. 515-530, Dec. 2003.

OLWEUS, Dan. Bullying in school: What we know and what we can do. Oxford: Blackwell, 1993. 140 p.

ORTEGA, Rosario; CALMAESTRA, Juan; MORA-MERCHÁN, Joaquín. Cuestinario Cyberbullying, 2007. Disponível em: <http://www.laecovi.es/ documents/CiberbullyingCuestionario.pdf> Acesso em: 10 Mar. 2012.

ORTEGA, Rosario; ELIPE, Paz; CALMAESTRE, José. Emociones de agresores y víctimas de cyberbullying: un estúdio preliminar en estudantes de Secundaria. Ansiedad y Estréss, Madrid, v. 15, n. 2-3, p. 151-165, dez. 2009.

ORTEGA, Rosario et al. The emotional impact of bullying and cyberbullying on victims: A European cross-national study. Aggressive Behavior, Malden, v. 38, n.5, p. 342-356, Sep./Oct. 2012.

PATCHIN, Justin; HINDUJA, Sameer. Cyberbullying and self-esteem. Journal of School Health, Malden, v. 80, n. 12, p. 614-621, Dec. 2010.

PEKRUN, Reinhard; FRENZEL, Anne C.; GOETZ, Thomas. The control-value theory of achievement emotions: An integrative approach to emotions in education. In: SCHUTZ, Paul A.; PEKRUN, Reinhard (Ed.). Emotion in education. Amsterdam: Academic Press. 2007. p. 13-36

PERREN, Sonja et al. Moral reasoning and emotion attributions of adolescent bullies, victims, and bully-victims. British Journal of Developmental Psychology, Malden, v. 30, n. 4, p. 511-530, Nov. 2012.

PESSOA, Teresa; AMADO, João. Cyberbullying - questões e desafios atuais. Edmetic: Revista de Educación Mediática e TIC, no prelo.

SAARNI, Carolyn. Emotional competence. A developmental perspetive. In: BAR-ON, Reuven; PARKER, James D. A. (Ed.). The handbook of emotional intelligence. San Francisco: Jossey Bass, 2000. p .68-91.

SAHIN, Mustafa; AYDIN, Betül; SARI, Serkan Volkan. Cyberbullying, cyber victimization and psychological symptoms: a study in adolescentes. Cukurova University Faculty of Education Journal, v. 41, n. 1, p. 53-59, 2012. Disponível em: <http://egitim.cu.edu.tr/efdergi>. Acesso em: 29 jun. 2013.

SMITH, Peter; BRAIN, Paul. Bullying in schools: lessons from two decades of research. Aggressive Behavior, Malden, v. 26, n. 1, p. 1-9, 2000.

SOUZA, Sidclay Bezerra; VEIGA SIMÃO, Ana Margarida; CAETANO, Ana Paula. Cyberbullying: perspetivas acerca do fenômeno e das estratégias de enfrentamento. Revista Psicologia: Reflexão e Crítica, v. 27, n. 3, p.582-590, 2014.

TTOFI, Maria; FARRINGTON, David. Reintegrative shaming theory, moral emotions and bullying behavior. Aggressive Behavior, Malden, v. 34, n. 4, p. 352-368, July/Aug. 2008.

TOPPING, Keith; HOLMES, Elisabeth; BREMNER, William. The effectiveness of school-based programs for the promotion of social competence. In: BAR-ON, Reuven; PARKER, James D. A. (Ed.). The handbook of emotional intelligence. San Francisco: Jossey Bass, 2000. p. 411-432 
WEINER, Bernard. Examining emotional diversity in the classroom: An attribution theorist considers the moral wmotions. In: SCHUTZ, Paul A.; PEKRUN, Reinhard (Ed.). Emotion in education. Amsterdam: Academic Press, 2007. p. 75-88.

WILTON, Melissa Mahadi; CRAIG, Wendy; PEPLER, Debra. Emotional regulation and display in classroom victims of bullying: characteristic expressions of affect, coping styles and relevant contextual factors. Social Development, Malden, v. 9, n. 2, p. 226- 245, May 2000.

Recebido em: 23.07.2014

Aprovado em: 14.03.2015

Ana Paula Caetano é professora associada do Instituto de Educação da Universidade de Lisboa, Portugal.

Isabel Freire é professora associada do Instituto de Educação da Universidade de Lisboa, Portugal.

Ana Margaria Veiga Simão é professora associada com agregação da Faculdade de Psicologia da Universidade de Lisboa, Portugal.

Maria José D. Martins é professora coordenadora da Escola Superior de Educação do Instituto Politécnico de Portalegre, Portugal.

Maria Teresa Pessoa é professora associada da Faculdade de Psicologia e de Ciências da Educação da Universidade de Coimbra, Portugal. 